\title{
Combined Application of Anticancer Vaccines of IEPOR Series and Doxorubicin in Rats with Transplanted Walker Carcinosarcoma
}

\author{
Olena Kruts ${ }^{1}$, Volodymyr Konovalenko ${ }^{2}$, Volodymyr Bazas ${ }^{3}$, Sergii Konovalenko ${ }^{2,}$, \\ Gennadii Didenko ${ }^{1}$, Oleksandr Lytvynenko ${ }^{4}$, Anna Artamonova ${ }^{5}$, Oksana Gerashchenko ${ }^{3}$ \\ ${ }^{1}$ Laboratory of Oncoimmunology and Design of Antitumor Vaccines, R. E. Kavetsky Institute of Experimental Pathology, Oncology and \\ Radiobiology, Kyiv, Ukraine \\ ${ }^{2}$ Tumor Monitoring and Therapy Design Department, R. E. Kavetsky Institute of Experimental Pathology, Oncology and Radiobiology, Kyiv, \\ Ukraine \\ ${ }^{3}$ Surgery Department, Kyiv City Oncology Center, Kyiv, Ukraine \\ ${ }^{4}$ Department of Skin and Soft Tissue Tumors, Institute of Cancer of the Ministry of Health of Ukraine, Kyiv, Ukraine \\ ${ }^{5}$ Department of Pharmacological and Biological Chemistry and Pharmacognosy, Kyiv Medical University, Kyiv, Ukraine
}

Email address:

servlakon@ukr.net (S. Konovalenko)

${ }^{*}$ Corresponding author

\section{To cite this article:}

Olena Kruts, Volodymyr Konovalenko, Volodymyr Bazas, Sergii Konovalenko, Gennadii Didenko, Oleksandr Lytvynenko, Anna Artamonova, Oksana Gerashchenko. Combined Application of Anticancer Vaccines of IEPOR Series and Doxorubicin in Rats with Transplanted Walker Carcinosarcoma. Journal of Cancer Treatment and Research. Vol. 8, No. 4, 2020, pp. 74-78.

doi: $10.11648 /$ j.jctr.20200804.12

Received: October 26, 2020; Accepted: November 11, 2020; Published: December 4, 2020

\begin{abstract}
Anticancer xenogeneic vaccine - is an agent, containing antigens of embryonic origin that underwent biotransformation under the action of cytotoxic proteins of B. subtilis B-7025. Anticancer efficacy of the vaccine is implemented by breaking immune system tolerance to own tumor antigens due to the antigenic similarity between tumor and embryonic proteins. The experiments were conducted in Wistar female rats (age 2.5 months and weight 200-220 g, bred at the animal house of R. E. Kavetsky Institute of Experimental Pathology, Oncology and Radiobiology). The care and use of the experimental animals have been performed in accordance with generally accepted international rules for conducting experiments in experimental animals. As an experimental tumor model, we have used Walker carcinosarcoma. In preclinical and clinical trials, success has been demonstrated in the use of a combination of anticancer vaccines with chemotherapy to achieve a synergistic effect, even if the dose and schedule of administration of the agents needed to be optimized. It has been shown that some drugs (doxorubicin, cyclophosphamide, docetaxel) induce immunological death of tumor cells, increase the expression of tumor-associated antigens, HLA-peptide complexes, thus sensitizing the tumor in vaccine-induced T-cell killing. It was determined that simultaneous administration of anticancer vaccines (regardless of the antigenic composition) and Doxorubicin resulted in a significant increase of survival and average lifespan of the experimental animals. The treated animals at the end of the experiment presented with increased cytotoxicity of lymphocytes and macrophages (both direct and antibodydependent), suggesting a reduced level of immunosuppression in experimental animals. In the group of rats, receiving Dox, the serum had no effect on the activity of lymphocytes. These data suggest that during the development of tumor the serum accumulates humoral factors, capable of blocking lymphocyte activity. Yet, as a result of additional activation (due to anticancer vaccines), the conditions are provided when the inhibitory activity of humoral factors is eliminated. The combined application of chemo- and biotherapy based on anticancer vaccines of IEPOR series is an efficient and rather perspective method of inhibition of malignant tumor process. The optimal scheme of the combined therapy was developed that involved the administration of anticancer vaccines together with the application of chemotherapeutic agents. The augmentation of antitumor effect can be explained by the reduction of immunosuppressive activity of blood serum towards the effector cells of antitumor immunity, resulting from the additional signal to the immune system - use of anticancer vaccines.
\end{abstract}


Keywords: Xenogeneic Anticancer Vaccine, Antimetastatic Efficiency, Combined Treatment

\section{Introduction}

In modern immunology and oncology, research and development in the field of anticancer vaccines, the study of their clinical effectiveness remains one of the most progressive directions. [1, 2] A priority focus area of modern medical science is, indeed, the development of novel approaches to the application of anticancer vaccines in combined administration with chemotherapeutic agents to improve the quality of life of cancer patients. Recently, more and more information on various variants of combined application of chemo- and biotherapeutic agents in oncology becomes available. Such a strategy allows minimization of toxic action on the immune system due to the reduction of the level of systemic immunosuppression and to create an effect of double blockage of tumor growth. Preclinical and clinical studies already have demonstrated the success of the application of combinations of anticancer vaccines (ACV) with chemotherapy and achieving of synergetic effect even in the case when optimization was required for the dose and regimen [3-6]. This was associated, first, with the possibility of elimination of immunosuppressive factors under the effect of chemotherapeutic agents, and second, with the induction of, so-called, immunogenic death of tumor cells $[7-9,10,12]$. It was demonstrated that chemotherapy is able to regulate the levels of co-stimulatory molecules (B7-1) or pathological coinhibitory molecules (PD-L1/B7-H1 or B7-H4), expressing on the surface of tumor cell; these mechanisms increase immune response effectiveness. [11]. In addition, due to chemotherapy, it is possible to increase the sensitivity of tumor cells to T-cell mediated lysis through Fas-, perforin- тa granzyme-B-dependent mechanisms [12]. Chemotherapeutic agents (in particular, anthracyclines) stimulate CCL2/CCR2dependent recruitment of functional antigen-presenting cells into tumor microenvironment, but not into lymph nodes [13]. In the clinical study M. von Mehren et. al [14] demonstrated that the patients with prior chemotherapy became more sensitive to ACV effect. It is believed that the much better result is determined by the vaccination of the patients with reduced tumor volume due to chemotherapy agent action [15].

The examples above substantiate the necessity of the combined application of chemo- and biotherapy. Therefore the aim of this study was to evaluate the efficacy of combined application of anticancer vaccines of IEPOR series and Doxorubicin in the rats with transplanted Walker carcinosarcoma.

At present, two types of vaccines have been developed in the Institute.

Anticancer autologous vaccine - an agent for personalized treatment of oncological patients that is used after total removal of tumor mass from a patient's body, aiming at prevention of recurrences and metastases. This original preparation contains tumor-associated antigens of autologous tissue material of a patient's tumor that underwent biotransformation under the action of cytotoxic lectin of B. subtilis B-7025. Antitumor and antimetastatic efficacy of the vaccine is implemented by breaking immune system tolerance to own tumor antigens. The introduction of anticancer autovaccine into the treatment regimens for oncological patients allows preventing the development of recurrences and metastases, increasing survival rates and improving their quality of life. The results of the clinical efficacy of anticancer autovaccine are protected with patents [16], and presented in the numerous publications.

Anticancer xenogeneic vaccine - is an agent, containing antigens of embryonic origin that underwent biotransformation under the action of cytotoxic proteins of $B$. subtilis B-7025. Anticancer efficacy of the vaccine is implemented by breaking immune system tolerance to own tumor antigens due to the antigenic similarity between tumor and embryonic proteins. Independent trial of this vaccine has demonstrated anticancer efficacy at its application in animals with tumor process [17]. Anticancer effect of the vaccine, as well, was also proved at the treatment of the patients with tumors of different genesis [18].

However, the question arises whether it is possible or reasonable to use these vaccines in combination with the modern chemotherapeutic agents. The answer to this question may be received only after the testing of these combinations in the animals with tumor process.

\section{Object and Methods of Research}

The experiments were conducted in Wistar female rats (age 2.5 months and weight 200-220 g, bred at the animal house of R. E. Kavetsky Institute of Experimental Pathology, Oncology and Radiobiology). The care and use of the experimental animals have been performed in accordance with generally accepted international rules for conducting experiments in experimental animals. As an experimental tumor model, we have used Walker carcinosarcoma.

Doxorubicin was injected to the study rats intraperitoneally, five times a day, every day, starting from the day 4 after transplantation of the tumor, at a dose of $0.25 \mathrm{mg}$ per animal that is $1.5 \mathrm{mg} / \mathrm{kg}$ of body mass. Anticancer vaccines of IEPOR series (autologous and xenogeneic embryonic vaccines) were injected subcutaneously by $0.5 \mathrm{ml}$ per injection by the following scheme: on days 5, 7 and 11 after tumor transplantation. The total dose of the vaccine per the whole course, equivalent to protein, was $1.5 \mathrm{mg}$ /animal.

The following experimental groups were formed: 1 tumor growth control (Control) — the animals with transplanted Walker tumor; 2 (Dox) - the animals with the tumor receiving Doxorubicin; 3 (XACV) - the animals with the tumor receiving xenogeneic embryonic anticancer vaccine; 4 (XACV + Dox) - the animals with the tumor receiving xenogeneic embryonic anticancer vaccine and 
Doxorubicin; 5 (AACV) - the animals with the tumor receiving autologous anticancer vaccine; 6 (AACV + Dox $)$ - the animals with the tumor receiving autologous anticancer vaccine and Doxorubicin.

During the experiment the following parameters were determined.

Tumor growth dynamics was evaluated by the change of the volume of a primary tumor lesion, the latter was determined by the formula: $\mathrm{V}=1 / 6 * \pi \mathrm{d}^{3}$, where $\mathrm{d}-$ is a diameter of the tumor nodule.

The survival of the animals in the experimental groups was evaluated by the percent of live animals in the groups of the total number of animals at the beginning of the experiment.

Average lifespan was assessed by the life duration of every animal in a group.

Cytotoxic activity (CTA) of lymphocytes (L), macrophages (M), and also antibody-dependent cytotoxicity (ADC) of the above effectors was evaluated in vitro with the MTT-test [23] on the day 23 after the SRT (surgical removal of tumor). Target cells were the cells of Walker carcinosarcoma. The blood serum (BS) effect was analyzed by potentiation index $(\mathrm{PI})$ :

$$
\mathrm{PI}=\left[\left(\mathrm{ADC}_{\mathrm{L} / \mathrm{M}}-\mathrm{CTA}_{\mathrm{L} / \mathrm{M}}\right) / \mathrm{CTA}_{\mathrm{L} / \mathrm{M}}\right] \cdot 100 \%
$$

In addition, the coefficient of neutrophil/lymphocyte ratio was analyzed - its increase is an evidence of systemic inflammatory processes and a factor of poor prognosis in such oncological diseases [19, 20].

The another evaluated factor was the ratio platelets-tolymphocytes; the reduction of this index is a marker of inflammatory processes intensity [21, 22.]. The statistic processing of the results was accomplished with generally accepted methods of variation statistics. Study results are presented as $\mathrm{M} \pm \mathrm{m}$, where $\mathrm{M}$ - is an arithmetic mean value, $\mathrm{m}$ - its standard error. The differences were accepted as reliable at $\mathrm{p}<0.05$. Calculations and plotting were done with application program OriginLab.

\section{Results and Discussion}

In the experiment, the significant antitumor activity of the combined action of anticancer vaccines of IEPOR series activity and preparation Doxorubicin was determined (Figure 1). Specifically, Dox or anticancer vaccines exerted a significant inhibitory effect on the development of tumor nodule in experimental animals as compared to the index of the control group.

The maximal accomplishment of antitumor effect was determined after the application of the combination of Dox and autologous anticancer vaccine. The apparent arrest of tumor growth was seen already from day 17 and up to the end of the experiment (day 36). Application of xenogeneic anticancer vaccine with Dox had a somewhat lower inhibitory effect, but it was higher than separate use of one or another vaccine. In the Doxorubicin group on day 25 the average volume of tumors was significantly lower. This was associated with mass mortality of animals in this group due to the large tumor size by that term of the experiment.

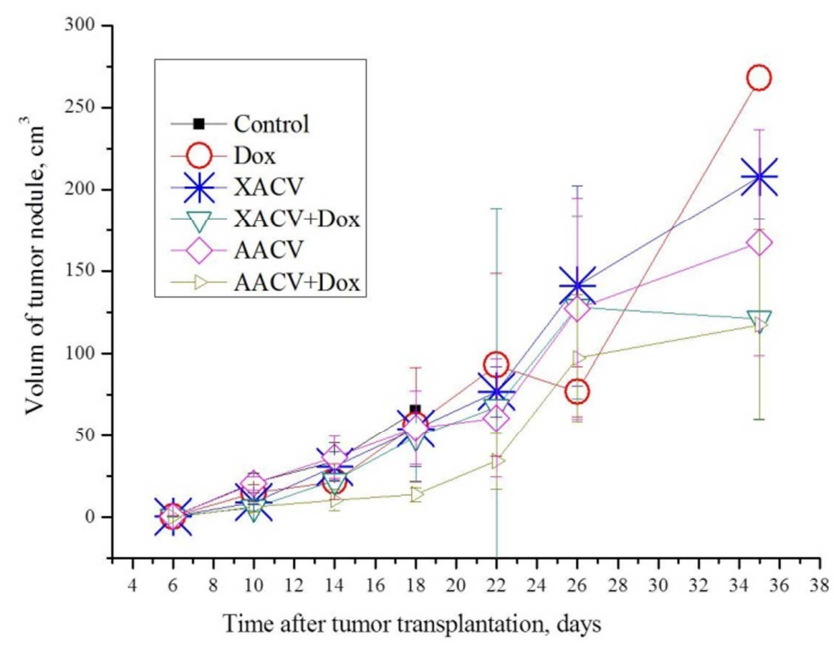

Figure 1. The dynamics of tumor growth of rats with Walker tumor who were administrated the autologous and xenogeneic vaccines.

The following survival rates were found at the end of the experiment (day 36): $0 \%$ - in tumor growth control, $20 \%$ - in the group, receiving Doxorubicin, $30 \%$ - in the group, receiving xenogenic anticancer vaccine; $40 \%$ - in animals, receiving autologous anticancer vaccine and combination of Doxorubicin with xenogeneic anticancer vaccine. The most efficient was the combination of autologous anticancer vaccine with Doxorubicin. The survival rate of this group was $50 \%$. (Figure 2)

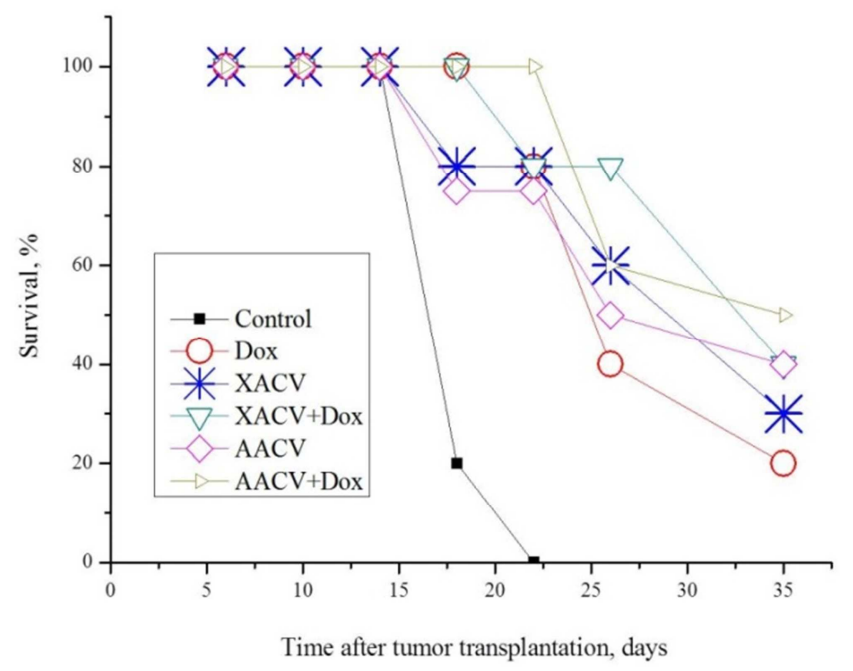

Figure 2. The survival of rats with Walker tumor who were administrated the autologous and xenogeneic vaccines.

Table 1. Average lifespan in the groups of animals.

\begin{tabular}{ll}
\hline Experimental groups & Average lifespan, days \\
\hline Control & $14.8 \pm 0.8$ \\
Dox & $24.6 \pm 2.89$ \\
XACV & $24.6 \pm 3.4$ \\
XACV+ Dox & $28 \pm 3.21$ \\
AACV & $26.4 \pm 4.01$ \\
AACV+ Dox & $28 \pm 2.95$ \\
\hline
\end{tabular}


The indices of the average lifespan in the groups of animals (Table 1), receiving simultaneously chemo- and immunotherapy, were maximal, regardless of antigenic composition of anticancer vaccine. The animals receiving monotherapy with either of the proposed approaches had a lower life duration.

Therefore, the optimal scheme was the combined application of anticancer vaccines (regardless of antigenic composition) with Doxorubicin. In this case, we can suggest the synergism of Dox and anticancer vaccines of IEPOR series.

The antitumor immune response is dependent upon the functioning of both specific (cytotoxic T-lymphocytes and plasmatic cells), and natural component of the immune system (macrophages). The application of the combined scheme in both cases has initiated the alterations in the immune status of the animals with tumor, in particular, it significantly led to the activation of cytotoxic activity of lymphocytes: cytotoxic index (CI) was 53.20 \pm 3.21 (AACV+Dox) and 45.15 \pm 4.37 (XACV+Dox), respectively, compared with intact animals or control rats $(34.80 \pm 3.15$ and $38.78 \pm 1.47 \%$ ). In the groups of animals, receiving Dox and $\mathrm{XACV}$, the activity of lymphocytes practically did not differ from the indices of the control group (Table 2).

Table 2. Cytotoxicity of lymphocytes in MTT-test in rats with surgically removed Lewis lung carcinoma that received either XACV and/or Dox.

\begin{tabular}{llll}
\hline Experimental groups & CTA L $(C I), \%$ & ADC L $(C I), \%$ & PI, \% \\
\hline Control & $38.78 \pm 1.47$ & $34.6 \pm 0.92$ & $-10,78$ \\
Dox & $37.28 \pm 1.33$ & $37.72 \pm 2.71$ & 1,18 \\
XACV & $38.17 \pm 1.67$ & $45.61 \pm 6.41$ & 19,49 \\
XACV+Dox & $45.15 \pm 4.37$ & $50.64 \pm 5.35 *$ & 12,15 \\
AACV & $44.38 \pm 3.73$ & $48.29 \pm 4.73$ & 8,81 \\
AACV+Dox & $52.17 \pm 3.49 *$ & $58.92 \pm 6.42 *$ & 12,94 \\
Intact animals & $34.80 \pm 3.15$ & $33.93 \pm 2.39$ & $-2,5$ \\
\hline
\end{tabular}

$* \mathrm{p}<0.05$ compared to the indices of control group (surgically removed tumor).

The addition of the autologous serum to the test (tumor cells and lymphocytes) inhibited the activity of lymphocytes in the control group. In all groups, receiving anticancer vaccines (regardless of their antigenic composition) the autologous serum increased the activity of lymphocytes. In the group of rats, receiving Dox, the serum had no effect on the activity of lymphocytes. These data suggest that during the development of tumor the serum accumulates humoral factors, capable of blocking lymphocyte activity. Yet, as a result of additional activation (due to anticancer vaccines), the conditions are provided when the inhibitory activity of humoral factors is eliminated.

The examination of the activity of the peritoneal macrophages in rats (Table 3) has demonstrated that the pattern of macrophage activation had a minor difference from the trends, determined in lymphocyte activation. And even addition of autologous serum to the test led to analogous outcomes as in adding of serum to the test with lymphocytes. These results suggest that in the organisms of the treated animals the general humoral background with the reduced immunosuppressive characteristics is formed.
Table 3. Cytotoxicity of macrophages in MTT-test in rats with transplanted Walker carcinosarcoma.

\begin{tabular}{llll}
\hline Experimental groups & CTA M $(C I), \%$ & ADC $(\mathbf{C I}), \%$ & PI, \% \\
\hline Control & $36.37 \pm 5.16$ & $34.53 \pm 3.76$ & $-5,06$ \\
Dox & $32.26 \pm 4.38$ & $32.71 \pm 2.96$ & 1,39 \\
XACV & $35.69 \pm 1.87$ & $37.69 \pm 2.22$ & 5,6 \\
XACV+Dox & $38.75 \pm 2.93$ & $39.38 \pm 4.21$ & 1,63 \\
AACV & $36.67 \pm 1.94$ & $39.02 \pm 3.90$ & 6,41 \\
AACV+Dox & $39.78 \pm 2.24$ & $42.18 \pm 3.33$ & 6,03 \\
Intact animals & $23.20 \pm 1.44$ & $21.16 \pm 1.91$ & $-8,79$ \\
\hline
\end{tabular}

\section{Conclusion}

Thereby, based on the study results, we can state that the combined application of chemo- and biotherapy based on anticancer vaccines of IEPOR series is an efficient and rather perspective method of inhibition of malignant tumor process. The optimal scheme of the combined therapy was developed that involved the administration of anticancer vaccines together with the application of chemotherapeutic agents. The augmentation of antitumor effect can be explained by the reduction of immunosuppressive activity of blood serum towards the effector cells of antitumor immunity, resulting from the additional signal to the immune system (use of anticancer vaccines). In the animals with the increased lifespan, the enhanced lymphocyte cytotoxicity (both direct and antibody-dependent) was seen. Moreover, in the animal groups with increased parameters of the average lifespan and survival rates the reduced amount of the circulating immune complexes that stimulate M-phenotype macrophage activation was found.

\section{References}

[1] Accolla RS, Buonaguro L, Melief C, Rammensee HG, Bassani-Sternberg M. Editorial: Novel Strategies for AntiTumor Vaccines. Front Immunol. 2020; 10: 3117. Published 2020 Jan 17.

[2] Charles J, Chaperot L, Hannani D et al. An innovative plasmacytoid dendritic cell line-based cancer vaccine primes and expands antitumor T-cells in melanoma patients in a firstin-human trial. Oncoimmunology. 2020 Apr 12; 9 (1): 1738812 .

[3] Guo C., Manjili M. H., Subjeck J. R. et al. (2013) Therapeutic cancer vaccines: past, present and future. Adv. Cancer Res., 119: 421-475.

[4] Zhao J., Huang Y., Liu D., Chen Y. (2014) Two hits are better than one: synergistic anticancer activity of $\alpha$-helical peptides and Doxorubicin/epirubicin. Oncotarget., 6 (3): 1769-1778.

[5] Sheng Sow H., Mattarollo S. R. (2013) Combining low-dose or metronomic chemotherapy with anticancer vaccines. A therapeutic opportunity for lymphomas. OncoImmunology, 2 (12): e27058.

[6] Iinuma H., Fukushima R., Inaba T. et al. (2014) Phase I clinical study of multiple epitope peptide vaccine combined with chemoradiation therapy in sophageal cancer patients. J. Transl. Med., 12: 84. 
[7] Gorbach O. I., Khranovska N. M., Skachkova O. V. et al. (2014) The chemoimmunotherapy based on dendritic cells and cisplatin in experiment. Biotechnol. Acta, 7 (4): 85-91.

[8] Ramakrishnan R., Assudani D., Nagaraj S. et al. (2010) Chemotherapy enhances tumor cell susceptibility to CTLmediated killing during cancer immunotherapy in mice. J. Clin. Invest., 120 (4): 1111-1124.

[9] Kim D., Monie A., Tsai Y-C. et al. (2008) Enhancement of CD4+ T-cell help reverses the Doxorubicin induced suppression of antigen-specific immune responses in vaccinated mice. Gene Ther., 15 (16): 1176-1183.

[10] Kepp O., Galluzzi L., Martins I. et al. (2011) Molecular determinants of immunogenic cell death elicited by anticancer chemotherapy. Cancer Metastasis Rev., 30: 61-69.

[11] Leisha A. Emens and Gary Middleton The Interplay of Immunotherapy and Chemotherapy: Harnessing Potential Synergies Cancer Immunology Research May 2015, 3 (5): 436-443 p.

[12] Singh et al., Differential dendritic cell-mediated activation and functions of invariant NKT-cell subsets in oral cancer Oral Dis. 2015 Jan; 21 (1): e105-13.

[13] Ma Y, Mattarollo et al., CCL2/CCR2-Dependent Recruitment of Functional Antigen Presenting Cells into Tumors upon Chemotherapy. Cancer Res. 2014 Jan 15; 74 (2): 436-45.

[14] von Mehren M., Arlen P., Gulley J. Et al. (2001) The influence of granulocyte macrophage colony-stimulating factor and prior chemotherapy on the immunological response to a vaccine (ALVAC-CEA B7.1) in patient with metastatic carcinoma. Clin. Cancer Res., 7: 1181-1191.

[15] Gulley J. L., Madan R. A., Schlom J. (2011) Impact of tumor volume on the potential efficacy of therapeutic vaccines. Current Oncol., 18: 150-157.

[16] Patent of Ukraine No. 57869. A method for producing of anticancer autovaccine. Potebnya G. P., Lisovenko G. S., Cheremshenko N. L., Tanasiienko O. A., Chekhun V. F.. (Ukraine) Appl. 15.06.2001; Publ. 15.07.2003; <Bull No. 7.
[17] J. Kraśko, K Zilionyte, A. Darinskas, N. Dobrovolskienė, A. Mlynska, S. Riabceva, I Zalutsky, Marina Derevyanko, V. Kulchitsky, O. Karaman, N. Fedosova, T. Symchych, G. Didenko, V. Chekhun, M. Strioga and V. Pašukonienè. / Post-operative unadjuvanted therapeutic xenovaccination with chicken whole embryo vaccine suppresses distant micrometastases and prolongs survival in a murine Lewis lung carcinoma model. Oncology Letters. V. 15: 2018. P. 50985104.

[18] Patent of Ukraine No. 73671 (2005). A method for producing of anticancer vaccine and method for oncological patients treatment / Potebnya G. P., Lisovenko G. S., Cheremshenko N. L., Semernikova L. I., Didenko G. V., Leshchenko Yu. M., Zavertylenko S. P., Chekhun V. F. (Ukraine); 15.08.2005, Bull. No. 8 .

[19] Walsh SR, Cook EJ, Goulder F, Justin TA, Keeling NJ Neutrophil-lymphocyte ratios as a prognostic factor in colorectal cancer. J Surg Oncol 91: 181-184.

[20] Keizman D, Gottfried M, Ish-Shalom M et al Pretreatment neutrophil-to-lymphocyte ratios in metastatic castration resistant prostate cancer patient treated with ketoconazole: association with outcome and predictive nomogram. Oncologist 17 (12): 1508-1514.

[21] Sunbul M, Gerin F, Durmus E, Kivrak T, Sari I, Tigen K, etal. Neutrophil to lymphocyte and platelet to lymphocyte ratios in patient with dipper versus non-dipper hypertension. Clin Exp Hypertens. 2014; 36 (4): 217-21.

[22] Seropian IM, Romeo FJ, Pizarro R, Vulcano NO, Posatini RA, Marenchino RG, Berrocal DH, Belziti CA Neutrophil-tolymphocyte ratios and platelet-to lymphocyte ratios as predictors of survival after heart transplantation. // ESC Hear tFail. 2018 Feb; 5 (1): 149-156.

[23] Ohno M., Abe T. (1991) Rapid colorimetric assay for the quantification of leukemia inhibitory factor (LIF) and interleukin-6 (IL-6). J. Immunol. Meth., 145: 199-203. 